\title{
Effects of Zuogui Wan and Yougui Wan on the Expression of Learning- and Memory-related Signal Transduction Molecules
}

\author{
Xiangping Kang ${ }^{1}$, Chao Liang ${ }^{2}$, Guoqin $\mathrm{Jin}^{1^{\star}}{ }^{*}$, Xueli Zhang ${ }^{1}$, Zhangbin Gong ${ }^{1}$, Yanwu Xu ${ }^{1}$ and Zhifen Han ${ }^{3}$ \\ ${ }^{1}$ Department of Biochemistry, Institute of Basic Medicine, Shanghai University of Traditional Chinese Medicine (TCM), Shanghai 201203, China \\ ${ }^{2}$ Experiment in TCM, Department, Institute of Basic Medicine, Shanghai University of Traditional Chinese Medicine (TCM), Shanghai 201203, China \\ ${ }^{3}$ Experiment Center for Teaching \& Learning, Shanghai University of Traditional Chinese Medicine (TCM), Shanghai 201203, China
}

"Corresponding author: Guoqin Jin, Department of Biochemistry, Institute of Basic Medicine, Shanghai University of Traditional Chinese Medicine (TCM), Shanghai 201203, China, Tel: +86-21-51322147; E-mail: jinguoqin8405@126.com

Received date: July 21, 2015, Accepted date: August 17, 2015, Published date: August 24, 2015

Copyright: $\odot 2015$, Kang X, et al. This is an open-access article distributed under the terms of the Creative Commons Attribution License, which permits unrestricted use, distribution, and reproduction in any medium, provided the original author and source are credited.

\begin{abstract}
Objective: This study aimed to determine the effect of kidney-tonifying recipe Zuogui Wan (ZGW) and Yougui Wan (YGW) on learning and memory ability, serum corticosterone (CORT), and expression of learning- and memory-related signal transduction molecules in aging rats.
\end{abstract}

Methods: Naturally aged rats (23 months) were used as animal model and were randomly divided into four groups: senile control group, senile+antagonist RU486 (antagonist of GR group, senile+ZGW group, and senile $+Y G W$ group). A fifth group was designated as the young group, composed of five-month old rats. Starting from the 21 st month, rats of senile+ZGW and senile+YGW group were administered with ZGW and YGW respectively for three months.

For subcutaneous injection, RU486 ( $5 \mathrm{mg} / \mathrm{kg}$ body weight) was administered $2 \mathrm{~h}$ prior to the Morris maze test (senile+antagonist RU486 group). Spatial learning and memory abilities were observed using Morris water maze test. Contents of serum CORT were examined using enzyme-linked immunosorbent assay.

Expression of related signal transduction molecules (CaM, pCaMK II, pERK1/2, pCREB, Arc, and pSynapsin I) were tested using Western blot. Regulating effects of kidney-tonifying recipe and RU486 on above-mentioned indicators were also studied.

Results: Contents of corticosterone in serum of senile rats were significantly higher compared with the young group. Morris water maze test showed that spatial learning and memory abilities of aging rats were clearly reduced. Expression of learning- and memory-related signal transduction molecules was also visibly lowered. However, kidney-tonifying recipe and RU486 exhibited potential to improve abnormal changes of the above-mentioned indicators in senile rats to varying extent.

Conclusion: Experimental findings indicate that kidney-tonifying recipe and RU486 may improve spatial learning and memory abilities and retard the effects of aging on senile rats by down-regulating abnormally high corticosterone levels and up-regulating expression of related signal transduction proteins.

Keywords: Aging; Corticosterone; Zuogui Wan; Yougui Wan; Learning and memory

\section{Research Highlights}

(1) This study was based on a theory of TCM: "Kidney Deficiency and Aging". Naturally aged rats were used as experimental animal models. Effects of kidney-tonifying recipes (Zuogui Wan and Yougui Wan) on degradation of learning and memory were determined. This research provides scientific experimental basis for clinical application of the two recipes. Furthermore, the present study may reveal scientific connotation of the theory: "Kidney Deficiency and Aging".

(2) Several studies have investigated the disorder in the hypothalamus-pituitary-adrenal (HPA) axis in aging rats. The mechanism by which high levels of corticosterone, which is the final hormone of HPA axis, eventually leads to degeneration of learning and memory remains unclear. In the present study, the molecular mechanism of age-related learning and memory impairment was observed, and the effect of kidney-tonifying recipes was also studied. These investigations were based on the learning- and memory-related signal transduction pathway, mediated by the glucocorticoid receptor (GR).

\section{Introduction}

Aging is a natural and complex biological process during which many physiological functions may become disordered, including impairment of learning and memory ability. Increasing amount of evidence have demonstrated that the hypothalamus pituitary-adrenal (HPA) axis is one of the most important neuroendocrine systems closely related to aging $[1,2]$. Disorder of HPA axis may cause damage 
to the hippocampus and degeneration of learning and memory ability, which can consequently destroy the steady state of the body and lead to aging [3].

The present research work revealed that the HPA axis in aging rats was hyperactive, leading to high levels of corticosterone which exhibit toxic effect on the hippocampus through the glucocorticoid receptor (GR) [4,5]. Hippocampus is part of the limbic system and is highly involved in learning and memory. The hippocampus is a GR-rich region and thus it is a key to the toxic effects of high levels of corticosterone (CORT) [6,7].

Therefore, the toxic effect of hippocampus may be fundamental to learning and memory degradation.

ZGW and YGW are two classic kidney-tonifying recipes prescribed by Jingyue Zhang of the Ming Dynasty. Both recipes exhibit effects such as anti-aging and enhancement of learning and memory ability [8]. In the present study, ZGW and YGW were administered to naturally aged rats.

Effects of ZGW and YGW on learning and memory ability, corticosterone level, and expression of learning- and memory-related signal transduction molecules (such as CaM, pCaMK II, pERK1/2, pCREB, Arc, and pSynapsin I) were observed.

Furthermore, the effects of the glucocorticoid receptor (GR) antagonist RU486 on the abovementioned indicators were also investigated. Results of the present study may provide scientific experimental basis for the application of ZGW and YGW on tonifying kidney and improving intelligence in clinical treatments.

\section{Material and Methods}

\section{Animals}

Male, pathogen-free grade Sprague-Dawley rats were provided by the Animal Laboratories in Shanghai University of Traditional Chinese Medicine, China (License No. SCXK2008-0016). Experimental use of animals complied with the Guidance Suggestions for the Care and Use of Laboratory Animals, issued by the Ministry of Science and Technology of China [9].

\section{Drugs}

RU486, 17-hydroxy-11-(4-dimethlaminophenyl)-17-(1-propynyl)estra-4,9-diene-3-one (purity $>98 \%$ ) was provided by Sigma-Aldrich Co., St. Louis, MO, USA. Structural formula of RU486 is shown in Figure 1.

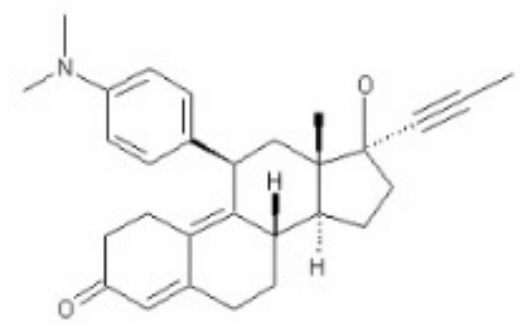

Figure 1: Structural formula of RU486.

\section{Chinese medicine compounds}

ZGW prescription: The decoction comprised Radix rehmanniae praeparata, Rhizoma dioscoreae, Semen cuscutae, Lycium barbarum L., Cornus officinalis, Radix achyranthis bidentatae, tortoise-plastron glue, and antler glue according to the ratio 4:2:2:2:2:1.5:2:2, immersed in eight times the volume of distilled water for $1 \mathrm{~h}$. The herbs were then decocted twice in simmer water for 30 minutes each time. The decoction was condensed to $1.53 \mathrm{~g}$ crude drug $/ \mathrm{ml}$ condensed decoction and was stored at $-20^{\circ} \mathrm{C}$ and rewarmed to $25^{\circ} \mathrm{C}$ to $30^{\circ} \mathrm{C}$ before administration.

YGW prescription: The decoction comprised Radix rehmanniae praeparata, Rhizoma dioscoreae, L. barbarum, C. officinalis, Semen cuscutae, antler glue, Eucommia ulmoides, Angelica sinensis, Cinnamomum cassia, and radix aconite according to the ratio 4:2:2:1.5:2:2:2:1.5:1:1, immersed in eight times the volume of distilled water for $1 \mathrm{~h}$. The herbs were then decocted twice in simmer water for 30 minutes each time.

The decoction was condensed to $1.83 \mathrm{~g}$ crude $\mathrm{drug} / \mathrm{ml}$ condensed decoction and was stored at $-20^{\circ} \mathrm{C}$ and rewarmed to $25^{\circ} \mathrm{C}$ to $30^{\circ} \mathrm{C}$ before administration. Drug doses: Doses of ZGW and YGW prepared were about 7 times the dose of adult body weight $(\mathrm{mg} / \mathrm{kg})[10,11]$. Rats were administered with $\mathrm{ZGW}$ and YGW respectively for 90 consecutive days starting from the 21st month [12].

\section{Establishment of aging rats and grouping}

One hundred and six 23-month old, specific pathogen-free grade, male Sprague-Dawley rats weighing $900 \mathrm{~g}$ to $1000 \mathrm{~g}$ were used in this study as aging rats. Twenty five-month-old, specific pathogen-free grade, male Sprague-Dawley rats weighing $300 \mathrm{~g}$ to $350 \mathrm{~g}$ were used as control rats (young rats).

Aging rats were randomly divided into the following four groups: senile control group (aged 23 months), senile+antagonist RU486 (aged 23 months), senile+ZGW group (aged 23 months), and senile+YGW group (aged 23 months). The fifth group was the young group (aged 5 months). Starting from age 21 months [12], the senile+ZGW group and senile+YGW group were administered with ZGW and YGW, respectively for 3 months.

\section{Systemic administration of RU486}

Steroid solutions were freshly prepared before injection by dissolving the crystalline steroid in absolute ethanol and dilution in polyethylene glycol (PEG) to a final ethanol concentration of $0.2 \%$. For subcutaneous injection, RU486 (5 $\mathrm{mg} / \mathrm{kg}$ body weight) was administered $2 \mathrm{~h}$ prior to the Morris maze test (senile+antagonist RU486 group) [13]. Rats of other groups received equal volumes of normal saline.

\section{Serum and brain tissue samples of rats}

Rats were euthanized under $10 \%$ chloral hydrate $(3.5 \mathrm{ml} / \mathrm{kg}$ weight) anesthesia, and $10-\mathrm{ml}$ blood samples were collected from the abdominal aorta using a syringe. Blood samples were then centrifuged at 3000 rounds per minute for 10 minutes at room temperature to obtain the serum. The brain was divided into two parts, namely, the cortex and the hippocampus. Serum and brain tissues were preserved in liquid nitrogen for 30 minutes and stored at $-70^{\circ} \mathrm{C}$. 
Page 3 of 6

\section{Morris water maze test [14]}

Five-day spatial navigation test was performed to detect average escape latency and to assess spatial learning and memory ability. Morris water maze equipment was provided by the Shanghai Geriatric Institute of Chinese Medicine. All rats were allowed to swim freely for 2 minutes a day prior to the test to adapt to the environment.

A certain amount of ink was poured into the swimming pool to hide the platform located at the first quadrant, $1.5 \mathrm{~cm}$ under the water. During the whole test, the platform was maintained in the same quadrant. Rats entered the water pool from the 4th quadrant.

A computer recorded the time the rats spent to find the platform (escape latency) and the swimming route of the rats. The time limit for each test was set at 70 seconds. Rats that failed to find the platform within the designated time limit were given 70 -second time records.

After test completion, rats were allowed to stay on the platform for 20 seconds. Each rat was subjected to the test twice a day, and average time of escape latency was calculated for further analysis.

\section{ELISA}

An enzyme-labeled immunosorbent assay (ELISA) kit was used to measure serum corticosterone levels in strict accordance with the manufacturer's instructions. Levels were calculated using a standard curve method.

\section{Western blot assay for CaM, pCaMK II, pERK1/2, pCREB, Arc and pSynapsin I expression in the hippocampus}

Total protein was extracted using RIPA lysis buffer (Beyotime, Shanghai, China), and total protein content was determined using a bicinchoninic acid quantitative protein detection kit (Beyotime, Shanghai, China).

Forty micrograms of hippocampus protein was mixed with sampling buffer solution and boiled in a water bath for 5 minutes. Four percent stacking gel and 10\% separation gel were prepared, and electrophoresis was performed under $60 \mathrm{~V}$ for 60 minutes and $100 \mathrm{~V}$ for about 100 minutes.

Extracted proteins were transferred to polyvinylidene fluoride membrane (Millipore Corporation) using a water-bath electric transfer device at $4^{\circ} \mathrm{C}, 350-\mathrm{mA}$ constant current for 80 minutes.
The membrane was blocked with $5 \%$ skimmed milk powder and shaken gently for $1 \mathrm{~h}$ at room temperature. After rinsing with TBST, membranes were incubated with rabbit anti-CaM, phosphor-CaMK II, phosphor-ERK1/2, phosphor-CREB, phosphor-Synapsin I, GAPDH polyclonal antibodies (1:500; Cell Signaling Technology), and mouse anti-Arc monoclonal antibody (1:200; Santa Cruz) overnight at $4^{\circ} \mathrm{C}$.

The membrane was rinsed with TBST four times for 10 minutes each time, and was then incubated with peroxidase conjugated goat anti-rabbit/mouse IgG (1:2000; Santa Cruz) at $37^{\circ} \mathrm{C}$ for 45 minutes and rinsed with TBST four times for 10 minutes each time.

Following polyvinylidene fluoride membrane coloration, Pierce chemiluminescent substrate solutions $\mathrm{A}$ and $\mathrm{B}$ were mixed for 5 minutes at room temperature and the scanned image was analyzed with a digital gel image analysis system (Protein Simple Company, USA).

\section{Statistical analysis}

Measurement data were expressed as mean \pm SD using SPSS16.0 software (SPSS, Chicago, IL, USA). Differences between groups were compared using one-way analysis of variance. Data of the water maze test were analyzed using analysis of variance of repeated data measurement [15].

For all statistical comparisons, a value of $\mathrm{P}<0.05$ was considered statistically significant.

\section{Results}

\section{Learning and memory abilities of experimental animals}

Morris test showed that latency shortened from the 1st day to the 5 th day of observation. Compared with the young rats, latency of the senile rats clearly increased from the 2 nd day $(\mathrm{P}<0.05)$.

Compared with the senile rats, latency of the senile+ZGW group shortened from the 2 nd day $(\mathrm{P}<0.05)$, and latency of the senile+YGW group and senile+antagonist RU486 group shortened from the 3rd day $(\mathrm{P}<0.05)$ (Table 1 and Figure 2).

\begin{tabular}{|c|c|c|c|c|c|}
\hline \multirow[t]{2}{*}{ Group } & \multicolumn{5}{|c|}{ Latency (second) } \\
\hline & 1st day & 2nd day & 3rd day & 4th day & 5th day \\
\hline Young group & $64.5 \pm 7.34$ & $55.7 \pm 9.37$ & $42.3 \pm 8.49$ & $33.6 \pm 7.52$ & $24.7 \pm 4.97$ \\
\hline Senile control group & $70.0 \pm 0.00$ & $68.8 \pm 2.57^{*}$ & $65.0 \pm 6.11^{*}$ & $63.0 \pm 6.06^{*}$ & $60.4 \pm 7.04^{*}$ \\
\hline Senile+antagonist RU486 group & $68.1 \pm 4.33$ & $61.2 \pm 7.51$ & $53.4 \pm 5.95 \Delta$ & $46.6 \pm 3.86 \Delta$ & $42.2 \pm 3.55 \Delta$ \\
\hline Senile+ZGW group & $67.1 \pm 4.50$ & $58.8 \pm 8.38 \Delta$ & $51.6 \pm 6.38 \Delta$ & $45.3 \pm 4.83 \Delta$ & $39.8 \pm 3.29 \Delta$ \\
\hline Senile+YGW group & $68.9 \pm 2.33$ & $60.2 \pm 8.39$ & $49.5 \pm 3.37 \Delta$ & $42.7 \pm 3.23 \Delta$ & $39.7 \pm 2.50 \Delta$ \\
\hline
\end{tabular}

Table 1: Comparison of spatial learning and memory ability in different groups. 
Citation: Kang X, Liang C, Jin G, Zhang X, Gong Z, et al. (2015) Effects of Zuogui Wan and Yougui Wan on the Expression of Learning- and Memory-related Signal Transduction Molecules. Altern Integr Med 4: 196. doi:10.4172/2327-5162.1000196

Page 4 of 6

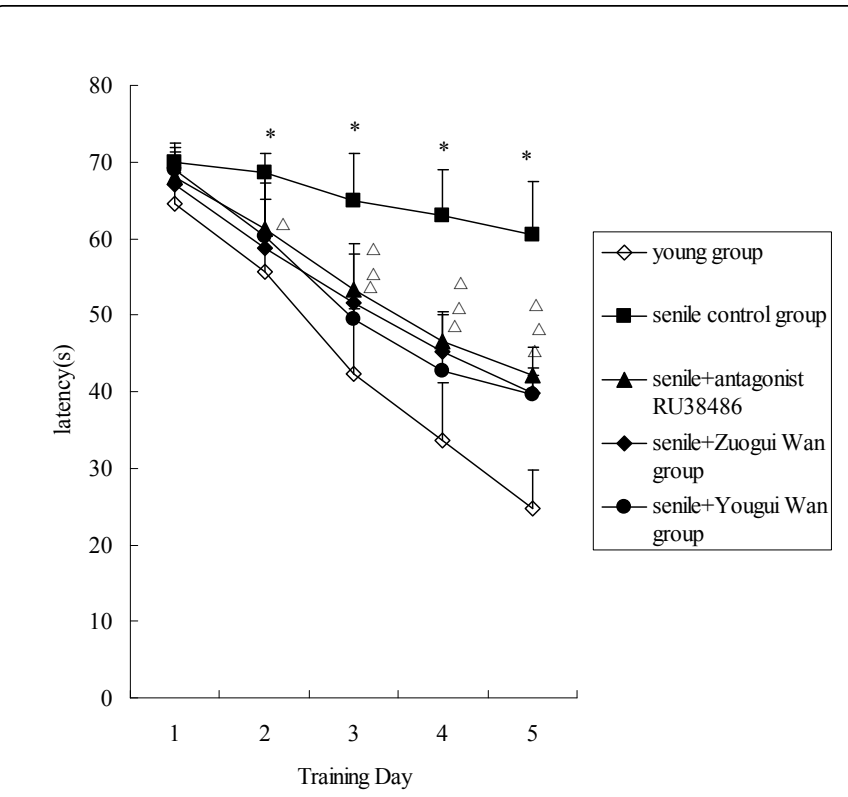

Figure 2: Escape latency (s) of experimental rats in Morris water maze test. ${ }^{\star} \mathrm{P}<0.05$, vs. young group, $\Delta \mathrm{P}<0.05$, vs. senile control group. Data are expressed as mean $\pm \mathrm{SD}, \mathrm{n}=10$ for each group. Variance analysis of repeated data measurement was used for comparison of several groups [15].

\section{Serum levels of corticosterone}

Results of the ELISA test demonstrated that corticosterone level in senile rats was significantly higher compared with the young rats group $(\mathrm{P}<0.05)$.

Compared with senile control group, content of corticosterone in senile+antagonist RU486 and senile+Zuogui Wan group clearly decreased $(\mathrm{P}<0.05)$.

The same trend was observed in senile+Yougui Wan group but the difference was not statistically significant (Table 2 and Figure 3).

\begin{tabular}{|l|l|}
\hline Group & CORT level (ng/L) \\
\hline young group & $71.89 \pm 7.63$ \\
\hline Senile control group & $195.13 \pm 10.80^{*}$ \\
\hline Senile+antagonist RU486 group & $82.55 \pm 7.71 \Delta$ \\
\hline Senile+ZGW group & $141.61 \pm 8.86 \Delta$ \\
\hline Senile+YGW group & $141.59 \pm 15.29$ \\
\hline
\end{tabular}

Table 2: Changes of CORT level in serum of different groups. ${ }^{\star} \mathrm{P}<0.05$, vs. young group, $\Delta \mathrm{P}<0.05$, vs. senile control group.

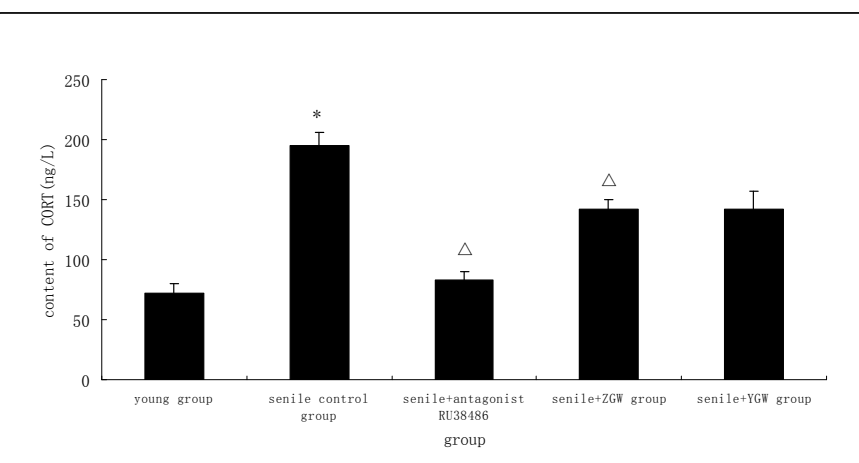

Figure 3: Changes of CORT level in serum of different groups. ${ }^{\star} \mathrm{P}<0.05$, vs. young group, $\Delta \mathrm{P}<0.05$, vs. senile control group. Data are expressed as mean $\pm \mathrm{SD}, \mathrm{n}=8$ for each group, using one-way analysis of variance and the Student-Newman-Keuls test. CORT: corticosterone.

\section{CaM, pCaMK II, pERK1/2, pCREB, Arc and pSynapsin I expression in rat hippocampus}

Semi-quantitative analysis using Western blot assay showed that CaM, pCaMK II, pERK1/2, pCREB, Arc, and pSynapsin I expression in the senile control group was significantly lower compared with the young group $(\mathrm{P}<0.05)$. Expression was increased to varying degrees by the kidney-tonifying recipe and RU486 (Table 3, Figures 4A and4B).

\begin{tabular}{|c|c|c|c|c|c|c|}
\hline Group & CaM & pCaMK II & pERK1/2 & pCREB & Arc & pSynapsin I \\
\hline young group & $0.578 \pm 0.075$ & $0.660 \pm 0.062$ & $0.613 \pm 0.061$ & $0.588 \pm 0.072$ & $0.568 \pm 0.067$ & $0.602 \pm 0.029$ \\
\hline Senile control group & $0.221 \pm 0.022^{*}$ & $0.250 \pm 0.029^{*}$ & $0.287 \pm 0.041^{*}$ & $0.185 \pm 0.032^{*}$ & $0.226 \pm 0.052^{*}$ & $0.206 \pm 0.022^{*}$ \\
\hline Senile+antagonist RU486 group & $0.409 \pm 0.055$ & $0.429 \pm 0.038$ & $0.432 \pm 0.045 \Delta$ & $0.371 \pm 0.040$ & $0.323 \pm 0.047$ & $0.391 \pm 0.041$ \\
\hline Senile+ZGW group & $0.444 \pm 0.073$ & $0.536 \pm 0.044 \Delta$ & $0.404 \pm 0.046$ & $0.426 \pm 0.049 \Delta$ & $0.328 \pm 0.045$ & $0.378 \pm 0.048$ \\
\hline Senile+YGW group & $0.398 \pm 0.03 \Delta$ & $0.515 \pm 0.047 \Delta$ & $0.440 \pm 0.036 \Delta$ & $0.421 \pm 0.049 \Delta$ & $0.327 \pm 0.062$ & $0.307 \pm 0.043$ \\
\hline
\end{tabular}

Absorbance ratios of CaM, pCaMK II, pERK1/2, pCREB, Arc, and pSynapsin I to GAPDH are expressed as mean \pm SD, $n=6$ for each group, using one-way analysis of variance and the Student-Newman-Keuls test. CaM: calmodulin; CaMK II: calmodulin-dependent protein kinase II; ERK: extracellular signal-regulated kinase; CREB: cAMP response-element binding protein; GAPDH: glyceraldehyde-3-phosphate dehydrogenase; ${ }^{*} \mathrm{P}<0.05$, vs. young group, $\Delta \mathrm{P}<0.05$, vs. senile control group.

Table 3: CaM, pCaMK II, pERK1/2, pCREB, Arc and pSynapsin I protein expression in hippocampus. 


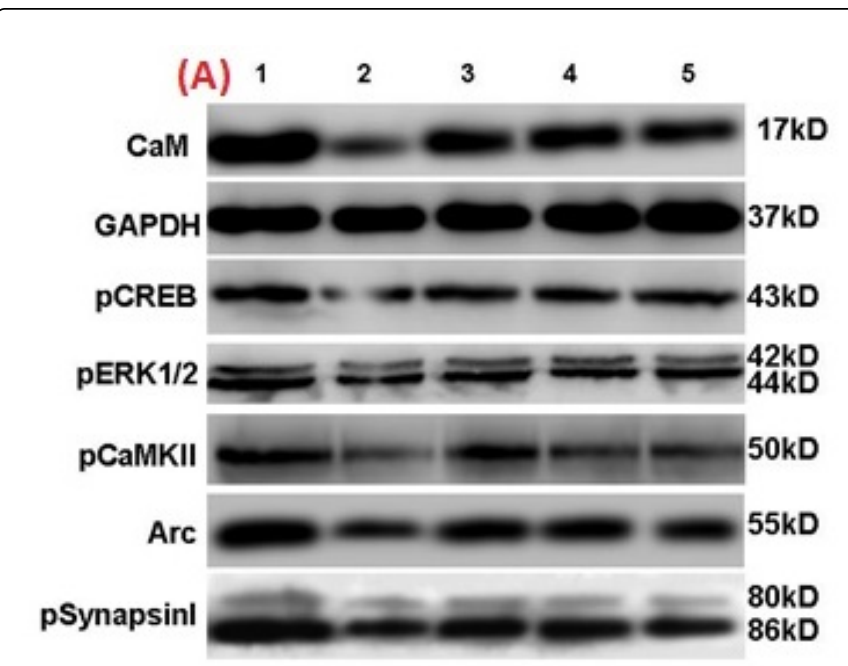

Figure 4A: CaM, pCaMK II, pERK1/2, pCREB, Arc and pSynapsin I expression in the hippocampus. Representative protein bands from Western blot for CaM, pCaMK II, pERK1/2, pCREB, Arc and pSynapsin I in hippocampus. 1-young group, 2-senile control group, 3-senile+antagonist RU486 group, 4-senile+ZGW group, 5senile+YGW group.

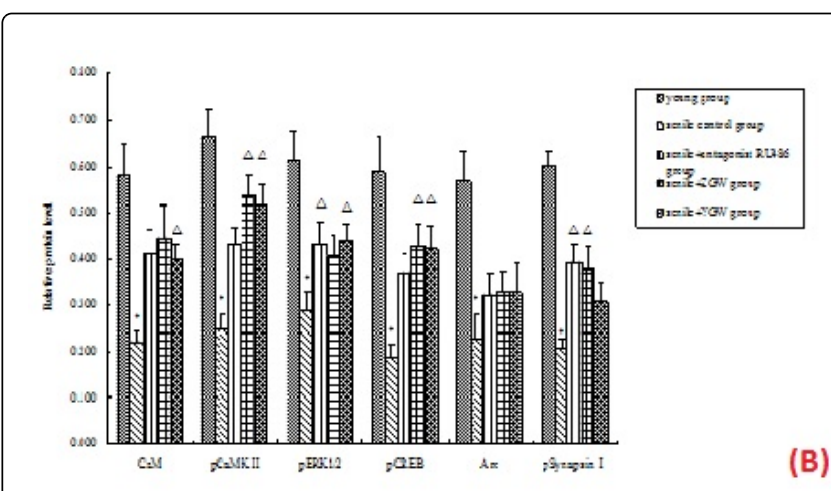

Figure 4B: CaM, pCaMK II, pERK1/2, pCREB, Arc and pSynapsin I expression in the hippocampus. Density values ratio of CaM, pCaMK II, pERK1/2, pCREB, Arc and pSynapsin I to GAPDH. Data are expressed as mean $\pm S D, n=6$ for each group, using oneway analysis of variance and the Student-Newman-Keuls test. ${ }^{\star} \mathrm{P}<0.05$, vs. young group, $\Delta \mathrm{P}<0.05$, vs. senile control group.

\section{Discussion}

Aging and age-associated cognitive impairments are complex and multifactorial processes. The process of normal aging often results in cognitive decline both in human and animal models. Growing evidence have shown that memory loss with aging may be associated with high levels of glucocorticoid [16,17]. Disorder in the HPA axis can be observed in humans and rodents during the normal process of aging, such as hypersecretion of glucocorticoid. High level of glucocorticoid is related to the action of the glucocorticoid receptor (GR) [18]. The GR is a nuclear receptor abundant in the hippocampus. High levels of glucocorticoid can cause atrophy of the hippocampus and decay of memory, which is mediated by GR [19]. Results of the present study showed that corticosterone levels in aging rats were significantly higher compared with the young rats, and the high level of corticosterone led to a decrease in the expression of GR in CA1 and CA2-3 subregion of hippocampus [20]. The abnormal changes caused hyperactivity in the HPA axis, resulting to the dysfunction of learning and memory. This response may be a form of self-defense of the neurons of hippocampus to the toxic effect of corticosterone.

Calmodulin-dependent protein kinase II (CaMK II) is a kind of serine/threonine protein kinase and is regulated by $\mathrm{Ca}^{2+} /$ calmodulin (CaM). Many studies have shown that CaMK II is related to synaptic plasticity and memory formation [21,22]. Furthermore, the function of CaMK II in reducing long term potentiation (LTP) of the hippocampus might be relevant to extracellular signal-regulated kinase (ERK), another key molecule in memory formation [23,24]. Regulation of the mitogen-activated protein kinase (MAPK)/ERK signaling pathway activated many transcription factors, such as cAMP response-element binding protein (CREB) $[25,26]$. These transcription factors exhibit important functions in synaptic plasticity by regulating the expression of specific molecules, such as activity-regulated cytoskeleton-associated protein (Arc) and synapsin (Syn). Increasing evidence has indicated that Arc and Syn have important functions in refactoring cytoskeleton of neurons, which include establishing synaptic morphology, strengthening connections between neurons, and consolidating LTP $[27,28]$. Thus, Arc and Syn are key molecules that maintiain long-term memory.

In the present study, expression of learning- and memory-related signal transduction molecules (such as CaM, pCaMK II, pERK1/2, pCREB, Arc, and pSynapsin I) of senile rats was observed. Furthermore, effect of classic kidney-tonifying recipe (ZGW and YGW) and RU486 were also tested. Results showed that senile rats exhibited poor learning ability and high level of corticosterone. Expression of learning- and memory-related signal transduction molecules also decreased. The results suggested that high levels of corticosterone injured the hippocampus by reacting with GR. Thus, subsequent signaling pathway mediated by GR was impaired. As a result, expression of many key molecules associated with learning and memory were decreased. In addition, synaptic plasticity and establishment of LTP were also affected, which could partly explain the degradation of learning and memory ability of aging rats.

ZGW and YGW are two classic kidney-tonifying recipes that were prescribed according to "interdependence between yin and yang". Sun et al. (YEAR) suggested that ZGW inhibited apoptosis and maintained stability of the cell membrane through improving the activity of adenosine triphosphatase (ATPase) [29]. Ma et al. (YEAR) proved that YGW regulated expression of apoptosis-related genes, such as bax and bcl-2 in the CA1 subregion of hippocampus in aging rats [30]. Results of the present study also showed that Zuogui Wan and Yougui Wan could improve abnormal changes, such as high levels of corticosterone, poor learning and memory ability, and low expression of learning and memory-related molecules in senile rats to varying extent. Furthermore, GR antagonist RU486 also worked on these indicators. The abovementioned observations revealed that the kidney-tonifying recipes (ZGW and YGW) and RU486 might improve spatial learning and memory ability and retard aging of senile rats by regulating the function of HPA axis, down-regulating abnormally high level of corticosterone, and up-regulating expression of related signal transduction molecules. 
Citation: Kang X, Liang C, Jin G, Zhang X, Gong Z, et al. (2015) Effects of Zuogui Wan and Yougui Wan on the Expression of Learning- and Memory-related Signal Transduction Molecules. Altern Integr Med 4: 196. doi:10.4172/2327-5162.1000196

Page 6 of 6

\section{Acknowledgement}

This study was financially supported by the National Natural Science Foundation of China (No.30873317, 81473583), Shanghai Natural Science Foundation of China (No.15ZR1441400), the leading Academic Discipline Project of Shanghai Municipal Education Commission (No.J50301), and Scientific Program of Shanghai Education Commission (No.2012JW03).

\section{References}

1. Pulopulos MM, Hidalgo V, Almela M, Puig-Perez S, Villada C, et al. (2015) Acute stress and working memory in older people. Stress 18: 178-187.

2. Khemissi W, Farooq RK, Le Guisquet AM (2014) Dysregulation of the hypothalamus-pituitary-adrenal axis predicts some aspects of the behavioral response to chronic fluoxetine: association with hippocampal cell proliferation. Front Behav Neurosci 8: 340

3. Aguilera G (2011) HPA axis responsiveness to stress: implications for healthy aging. Exp Gerontol 46: 90-95.

4. Zhao XY, Jin GQ, Gu CY (2010) Effects of Zuogui Wan and Yougui Wan on learning and memory related signal transduction molecular mRNA expressions in the corticosterone induced rat's hippocampus neuron pathological model. China Journal of TCM and Pharmacy 25: 1983-1987

5. Dai WW, Jin GQ, Zhang XL (2004) Effects of kidney-tonifying herbs on the glucocorticoid receptor mRNA expression in hippocampal subfields of aging rats. Acta Biochimica et Biophyscia Sinica 36: 163

6. Hu P, Oomen C, van Dam AM, Wester J, Zhou JN, et al. (2012) A singleday treatment with mifepristone is sufficient to normalize chronic glucocorticoid induced suppression of hippocampal cell proliferation. PLoS One 7: e46224.

7. Chen KC, Blalock EM, Curran-Rauhut MA, Kadish I, Blalock SJ, et al. (2013) Glucocorticoid-dependent hippocampal transcriptome in male rats: pathway-specific alterations with aging. Endocrinology 154: 2807-2820.

8. Kang XP, Jin GQ, Liang C (2013) Effects of kidney-tonifying recipe on the expressions of learning and memory related signal molecules. Chinese Journal of Gerontology 33: 2064-2067

9. The Ministry of Science and Technology of the People's Republic of China. Guidance Suggestions for the Care and Use of Laboratory Animals 2006-09-30

10. Lu Y, Zhang DF (2012) Pharmacology of Chinese Materia Medica. Beijing: People's Medical Publication House.

11. Wei W, Wu XM, Li YJ (2010) Experimental Methodology of Pharmacology. Beijing: People's Medical Publication House.

12. Kang XP, Jin GQ, Gong ZB (2007) Effects of Zuogui Pill and Yougui Pill on the expression of amine acids neurotransmitter receptor in the aging rat's hypothalamus. Pharmacology and Clinics of Chinese Materia Medica 23: 6-8

13. Xu L, Holscher C, Anwyl R, Rowan MJ (1998) Glucocorticoid receptor and protein/RNA synthesis-dependent mechanisms underlie the control of synaptic plasticity by stress. Proc Natl Acad Sci U S A 95: 3204-3208.

14. Morris RJ (1984) Development of a water-maze procedure for studying spatial learning in the rats. Neurosci Methods 11: 47-60
15. Qiu H, Jin GQ, Jin RF, Zhao WK (2007) [Analysis of variance of repeated data measured by water maze with SPSS]. Zhong Xi Yi Jie He Xue Bao 5 101-105.

16. Hasan KM, Rahman MS, Arif KM, Sobhani ME (2012) Psychological stress and aging: role of glucocorticoids (GCs). Age (Dordr) 34: 1421-1433.

17. Liston C, Gan WB (2011) Glucocorticoids are critical regulators of dendritic spine development and plasticity in vivo. Proc Natl Acad Sci U S A 108: 16074-16079.

18. Pardon MC, Sarmad S, Rattray I (2009) Repeated novel cage exposure induced improvement of early Alzheimer's like cognitive and amyloid changes in TASTPM mice is unrelated to changes in brain endocannabinoids levels. Neurobiology Aging 30: 1099-1113

19. Jeanneteau F, Chao MV (2013) Are BDNF and glucocorticoid activities calibrated? Neuroscience 239: 173-195.

20. Kang XP, Jin GQ (2012) Degradation mechanisms of learning and memory in aging rats and the effects of kidney-tonifying recipe. China Journal of TCM and Pharmacy 27: 981-986

21. Uddin RK, Singh SM (2013) Hippocampal gene expression meta-analysis identifies aging and age-associated spatial learning impairment (ASLI) genes and pathways. PLoS One 8: e69768.

22. Karthik Bodhinathan, B.Tech, Ashok Kumar (2010) Intracellular redox state alters N-Methyl D-Aspartate receptor response during aging through $\mathrm{Ca} 2+/$ calmodulin -dependent protein kinase II. J Neurosci 30: 1914-1924

23. Steelman LS, Chappell WH, Abrams SL, Kempf RC, Long J, et al. (2011) Roles of the Raf/MEK/ERK and PI3K/PTEN/Akt/mTOR pathways in controlling growth and sensitivity to therapy-implications for cancer and aging. Aging (Albany NY) 3: 192-222.

24. Martinez-Lopez N, Athonvarangkul D, Mishall P, Sahu S, Singh R (2013) Autophagy proteins regulate ERK phosphorylation. Nat Commun 4: 2799.

25. Morris KA, Gold PE (2012) Age-related impairments in memory and in CREB and pCREB expression in hippocampus and amygdala following inhibitory avoidance training. Mech Ageing Dev 133: 291-299.

26. Hansen RT 3rd, Zhang HT (2013) Senescent-induced dysregulation of cAMP/CREB signaling and correlations with cognitive decline. Brain Res 1516: 93-109.

27. Sama DM, Norris CM (2013) Calcium dysregulation and neuroinflammation: discrete and integrated mechanisms for age-related synaptic dysfunction. Ageing Res Rev 12: 982-995.

28. Miyashita T, Kubik S, Lewandowski G, Guzowski JF (2008) Networks of neurons, networks of genes: an integrated view of memory consolidation. Neurobiol Learn Mem 89: 269-284.

29. Sun LL, Kang GS, Ma YC (2010) The effect of Zuogui pill on ATPase bcl-2 bax and caspase- 3 of subacute aging rats induced by D-galactose. Chinese Archives of Traditional Chinese Medicine 28: 205-207

30. Ma SJ, Cao S, Yao JP (2011) Effect of bushen prescription on expression of apoptosis related gene-bax, bcl-2 mRNA in hippocampal CAlof natural aged rats. Pharmacology and Clinics of Chinese Materia Medica $27: 6-7$ 